\title{
Generalized Foldy-Lax Formulation ${ }^{1}$
}

\author{
Kai Huang $^{2} \quad$ Knut Solna $^{3} \quad$ Hongkai Zhao $^{3}$
}

${ }^{1}$ The research is partially supported by ONR grant N00014-05-1-0442.

${ }^{2}$ Department of Mathematics, Florida International University, Miami, FL 33199

${ }^{3}$ Department of Mathematics, University of California at Irvine, Irvine, CA 92697-3875, KS was also partly supported by NSF grant DMS0709389. 


\begin{abstract}
We present a method for numerical wave propagation in a heterogeneous medium. The medium is defined in terms of an extended scatterer or target which is surrounded by many small scatterers. By extending the classic Foldy-Lax formulation we developed an efficient algorithm for numerical wave propagation in two dimension. In the method that we set forth multiple scattering among the point scatterers and the extended target is fully taken into account via a boundary integral formulation coupled with the FoldyLax formulation. This formulation forms the basis for our numerical procedure.
\end{abstract}


Keywords: Helmholtz equation, numerical wave propagation, Foldy-Lax, boundary integral equation, scattering.

\section{Introduction}

The original Foldy-Lax formulation gives a model for the scattered wave-field, at a particular frequency, in the case of a collection of point scatterers. In this idealized situation of a cluttered medium the scattered field derives from the solution of a linear system. Therefore, despite a very complicated scattering picture with multiples of all orders, the scattered field can be computed very effectively. In many situations one wishes however to simulate waves in a cluttered medium with an imbedded extended scatterer. This is for instance the case when one wants to use the numerical scheme for assessment of schemes for imaging in a cluttered environment. Then one typically needs to solve a forward problem corresponding to numerical wave propagation in the heterogeneous medium. This is a challenging task that in general involves phenomena on many scales, in particular the scales of the medium variations, the wave-length and the propagation distance. In the approach we take here the medium clutter is modeled by a set of point scatterers and the extended object by a connected region with a smooth boundary. We developed here an efficient numerical algorithm by extending the original Foldy-Lax formulation to this case with an embedded extended scatterer. We remark that in the motivating application to imaging it is important to resolve the geometry of the extended target and the effect it has on the scattered field. In the case with only an extended scatterer present the scattered field can be computed effectively using various numerical methods, while in the case of only point scatterers the Foldy-Lax method can be used. The challenge here is to combine methods such that the multiple scattering in between the extended scatterer and the multiple scatterers is taken into account, ideally without increasing significantly the computational complexity relative to the original algorithms. 
In our approach we use the extended boundary condition method (EBCM), a boundary integral formulation, to compute the scattering operator (matrix) associated with the extended target with Dirichlet boundary condition. The method can generalize to other boundary conditions without difficulty. Then, we derive a coupled linear system to capture the multiple scattering between the extended target and the collection of point scatterers. We consider the model problem in $2 \mathrm{D}$ only, it is possible to generalize to three dimension case.

A main aspect of our approach is that the free space Green function can be used for propagation in between the scatterers. As a consequence we do not have to solve any partial differential equation numerically and we can deal with long propagation distances and a large number of point scatterers, which is important when we are dealing with phenomena in the far field.

The outline of the paper is as follows. In Section 2 we briefly review the FoldyLax formation for point scatterers and in Section 3 the extended boundary condition method for extended targets. In Section 4 we present our approach which integrates the two methods. Finally, in Section 5, we present some numerical results. Throughout the paper we consider a scalar wave field in the frequency domain so that the governing equation is the Helmholtz equation. The problem set-up is illustrated in Figure 1. A time-harmonic source generates a wave that is impinging on a heterogeneous medium consisting of an extended scatterer embedded in a background medium of point scatterers. Our main objective is to compute the resulting scattered field.

\section{Foldy-Lax System for Point Scatterers}

We consider here a collection of $N$ point scatterers in a homogeneous medium and describe how the Foldy-Lax method can be used to model the scattered field, see $[5,6,7]$ for a more detailed description. Let $\phi_{i n c}(\mathbf{r})$ denote the incident wave-field 

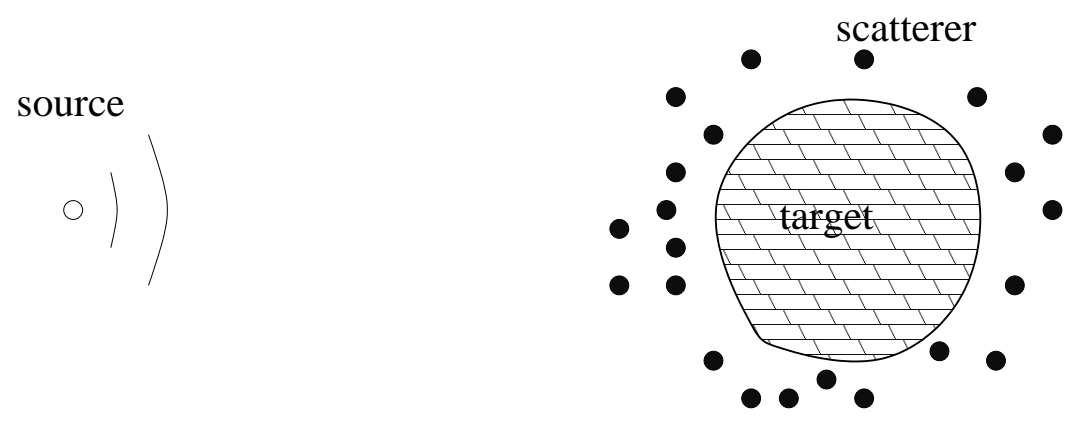

Figure 1: Physical setup of the problem. A source generates a wave that is impinging on a heterogeneous medium consisting of an extended scatterer embedded in a background medium of point scatterers.

generated by the source. Each scatterer will now have impinging upon it this incident wave in addition to the total scattered field from all the other scatterers.

Given a single scatterer at $\mathbf{r}_{j}$, and a field $\phi_{j}^{E}$ ( the exciting field ) impinging upon it, the scattered field from it and evaluated at $\mathbf{r}$ is then modeled by:

$$
\phi_{j, s c a}(\mathbf{r})=\sigma_{j} g\left(\mathbf{r}, \mathbf{r}_{\mathbf{j}}\right) \phi_{j}^{E},
$$

where $\sigma_{j}$ is the scattering amplitude and $g\left(\mathbf{r}, \mathbf{r}^{\prime}\right)$ is the free space Green function satisfying the Helmholtz equation:

$$
\left(\nabla^{2}+k^{2}\right) g\left(\mathbf{r}, \mathbf{r}^{\prime}\right)=-\delta\left(\mathbf{r}-\mathbf{r}^{\prime}\right) .
$$

In our notation, we suppress the dependence of the Green function on the wave number $k$. For $N$ point scatterers at $\mathbf{r}_{1}, \mathbf{r}_{2}, \cdots, \mathbf{r}_{N}$, the scattered field is given by:

$$
\phi_{s c a}(\mathbf{r})=\sum_{j=1}^{N} \sigma_{j} g\left(\mathbf{r}, \mathbf{r}_{j}\right) \phi_{j}^{E},
$$

where $\phi_{j}^{E}$ is the exciting field at point scatterer $\mathbf{r}_{j}$. The exciting fields at the point scatterers are now coupled via the linear system:

$$
\phi_{j}^{E}=\phi_{i n c}\left(\mathbf{r}_{j}\right)+\sum_{l=1, l \neq j}^{N} \sigma_{l} g\left(\mathbf{r}_{j}, \mathbf{r}_{l}\right) \phi_{l}^{E} .
$$


We rewrite (2) in the following form:

$$
\phi_{j}=b_{j}+\sum_{l=1, l \neq j}^{N} B_{j l} \phi_{l},
$$

where $\phi_{j}=\phi_{j}^{E}, B_{j l}=\sigma_{l} g\left(\mathbf{r}_{j}, \mathbf{r}_{l}\right)$ and $b_{j}=\phi_{i n c}\left(\mathbf{r}_{j}\right)$.

Define now the matrix $Z$ :

$$
Z_{i j}=\left\{\begin{array}{cc}
1, & \text { if } i=j \\
-B_{i j}, & \text { if } i \neq j
\end{array}\right.
$$

Then (2) is in the matrix form:

$$
\mathbf{Z} \phi=b,
$$

where $\phi$ and $b$ are vectors with $\phi_{l}$ and $b_{l}$ as components respectively. It is clear that $\mathbf{Z}$ is invertible for the $\sigma_{j}$ 's sufficiently small.

After solving the above linear system, we obtain the scattered field from (2).

\section{Extended Boundary Condition Method}

We introduce next the Extended Boundary Condition Method for computing the scattered field from an extended target in a homogeneous medium. We let $D$ be a bounded simply connected domain which define the support of the scatterer and we shall here consider the case with two space dimensions. The smooth boundary of $D$ is denoted $\mathbf{S}=\partial D$. We then consider the following scalar wave equation in the exterior of $D$ :

$$
\begin{aligned}
\left(\nabla^{2}+k^{2}\right) \phi(\mathbf{r}) & =q(\mathbf{r}) & & \text { in } R^{2} \backslash D, \\
\phi(\mathbf{r}) & =0 & & \text { on } \mathbf{S} .
\end{aligned}
$$

Note that we assume a Dirichlet boundary condition at the target. That is, for simplicity we choose here a sound-soft obstacle, however, the method we shall introduce generalizes to other boundary conditions. 
By applying Green's theorem to the above Helmholtz equation, we obtain the following boundary integral equations:

$$
\begin{aligned}
& \phi_{i n c}(\mathbf{r})-\int_{\mathbf{S}} d s^{\prime} \hat{n} \cdot\left[g\left(\mathbf{r}, \mathbf{r}^{\prime}\right) \nabla^{\prime} \phi\left(\mathbf{r}^{\prime}\right)\right]=0, \quad \mathbf{r} \in D, \\
& \phi_{i n c}(\mathbf{r})-\int_{\mathbf{S}} d s^{\prime} \hat{n} \cdot\left[g\left(\mathbf{r}, \mathbf{r}^{\prime}\right) \nabla^{\prime} \phi\left(\mathbf{r}^{\prime}\right)\right]=\phi(\mathbf{r}), \quad \mathbf{r} \in R^{2} \backslash D,
\end{aligned}
$$

where we have used the expression

$$
\phi_{i n c}(\mathbf{r})=-\int_{R^{2} \backslash D} d A^{\prime} g\left(\mathbf{r}, \mathbf{r}^{\prime}\right) q\left(\mathbf{r}^{\prime}\right),
$$

for the incoming field generated by the source, and $\hat{n}$ is the exterior unit normal direction. We can now first solve for $\hat{n} \cdot \nabla \phi$ on the boundary $\mathbf{S}$ from the equation (7), and then in a second step obtain the scattered field from (8).

The extended-boundary-condition method, developed by Waterman $[9,10]$ is an alternative to solving the above boundary integral equation. In this method, the integral equations are imposed not on the boundary $\mathbf{S}$, but on some extended boundary $\mathbf{S}_{1}$ and $\mathbf{S}_{2}$ away from $\mathbf{S}$. The boundary $\mathbf{S}_{2}$ is a circle inside of the domain $D$, while $\mathbf{S}_{1}$ is a circle which contains the region $D$. We choose the origin of the coordinate system to be inside of $\mathbf{S}_{2}$ such that $\left|\mathbf{r}_{\text {in }}\right|<|\mathbf{r}|<\left|\mathbf{r}_{\text {out }}\right|$, for $\mathbf{r}_{\text {in }} \in \mathbf{S}_{2}, \mathbf{r} \in \mathbf{S}, \mathbf{r}_{\text {out }} \in \mathbf{S}_{1}$. This configuration is illustrated in Figure 3.

In a homogeneous medium, by the additional theorem [4], the Helmholtz Green's function can be expanded as:

$$
g\left(\mathbf{r}, \mathbf{r}^{\prime}\right)=i \sum_{n=-\infty}^{\infty} S_{n}(\mathbf{r}) R_{n}\left(\mathbf{r}^{\prime}\right), \quad \text { for }|\mathbf{r}|>\left|\mathbf{r}^{\prime}\right|
$$

where $S_{n}$ represents an outgoing wave cylindrical harmonic, and $R_{n}$ is the real part of $S_{n}: R_{n}=\operatorname{Real} S_{n}$ 


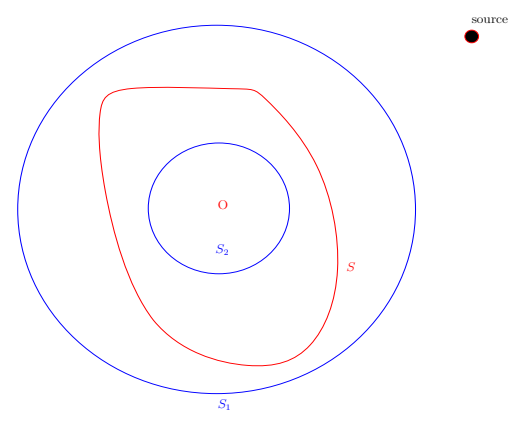

Figure 2: The figure shows the Physical Boundary, $S$, of the extended scatterer and the "Extended Boundary" $S_{1} \& S_{2}$.

We have explicitly, for $\mathbf{r}=(|\mathbf{r}|, \alpha)$ in polar coordinates

$$
\begin{aligned}
& n=0, \quad S_{0}(\mathbf{r})=H_{0}^{(1)}(k|\mathbf{r}|) / 2, \\
& R_{0}(\mathbf{r})=J_{0}(k|\mathbf{r}|) / 2, \\
& n<0, \quad S_{n}(\mathbf{r})=H_{n}^{(1)}(k|\mathbf{r}|) \cos (n \alpha) / \sqrt{2}, \\
& R_{n}(\mathbf{r})=J_{n}(k|\mathbf{r}|) \cos (n \alpha) / \sqrt{2}, \\
& n>0, \quad S_{n}(\mathbf{r})=H_{n}^{(1)}(k|\mathbf{r}|) \sin (n \alpha) / \sqrt{2}, \\
& R_{n}(\mathbf{r})=J_{n}(k|\mathbf{r}|) \sin (n \alpha) / \sqrt{2},
\end{aligned}
$$

where $H_{n}^{(1)}$ and $J_{n}$ are Hankel function and Bessel functions of the first kind respectively. Using reciprocity we find that the incident wave from a point source at $\mathbf{r}_{0}$ can be expanded as:

$$
\phi_{i n c}(\mathbf{r})=g\left(\mathbf{r}, \mathbf{r}_{0}\right)=i \sum_{n} S_{n}\left(\mathbf{r}_{0}\right) R_{n}(\mathbf{r}) \equiv \sum_{n} a_{n} R_{n}(\mathbf{r})
$$

so that

$$
a_{n}=i S_{n}\left(\mathbf{r}_{0}\right)
$$

We moreover expand the unknown on the boundary $\mathbf{S}$ as

$$
\hat{n} \cdot \nabla \phi(\mathbf{r})=\sum_{m} b_{m} \hat{n} \cdot \nabla R_{m}(\mathbf{r}), \quad \mathbf{r} \in S,
$$


We next describe the approach that gives these unknowns in terms of the incident wave. First, on the interior boundary $\mathbf{S}_{2}$, we substitute the above expansion into equation (7):

$$
\sum_{n} a_{n} R_{n}(\mathbf{r})=i \int_{\mathbf{S}} d s^{\prime} \sum_{m} b_{m} \hat{n} \cdot \nabla^{\prime} R_{m}\left(\mathbf{r}^{\prime}\right) \sum_{n} S_{n}\left(\mathbf{r}^{\prime}\right) R_{n}(\mathbf{r})
$$

From the orthogonality of the cylindrical harmonics, we then have that:

$$
\begin{aligned}
a_{n} & =i \sum_{m} b_{m} \int_{\mathbf{S}} d s^{\prime}\left[S_{n}\left(\mathbf{r}^{\prime}\right) \hat{n} \cdot \nabla^{\prime} R_{m}\left(\mathbf{r}^{\prime}\right)\right] \\
& \equiv i \sum_{m} b_{m} Q_{n m}
\end{aligned}
$$

where

$$
Q_{n m}=\int_{\mathbf{S}} d s^{\prime}\left[S_{n}\left(\mathbf{r}^{\prime}\right) \hat{n} \cdot \nabla^{\prime} R_{m}\left(\mathbf{r}^{\prime}\right)\right]
$$

Once the above linear system (12) is solved for $b_{m}$, the scattered field can be found by the following. On the exterior boundary $\mathbf{S}_{1}$, we write

$$
\begin{aligned}
\phi_{s c a}(\mathbf{r}) & =\phi(\mathbf{r})-\phi_{i n c}(\mathbf{r}) \\
& \equiv \sum_{n} f_{n} S_{n}(\mathbf{r}) .
\end{aligned}
$$

Then we readily deduce from (8) and (9) that

$$
\begin{aligned}
f_{n} & =-i \int_{\mathbf{S}} d s^{\prime} R_{n}\left(\mathbf{r}^{\prime}\right) \hat{n} \cdot\left[\sum_{m} b_{m} \nabla^{\prime} R_{m}\left(\mathbf{r}^{\prime}\right)\right] \\
& =-i \sum_{m} b_{m} \int_{\mathbf{S}} d s^{\prime}\left[R_{n}\left(\mathbf{r}^{\prime}\right) \hat{n} \cdot \nabla^{\prime} R_{m}\left(\mathbf{r}^{\prime}\right)\right] \\
& =-i \sum_{m} b_{m} \operatorname{Real}\left[Q_{n m}\right] .
\end{aligned}
$$

Consequently, we have

$$
\mathbf{a}=i \mathbf{Q} \cdot \mathbf{b}
$$




$$
\mathbf{f}=-i \operatorname{Real}[\mathbf{Q}] \cdot \mathbf{b}=-\operatorname{Real}[\mathbf{Q}] \cdot \mathbf{Q}^{-1} \cdot \mathbf{a}
$$

where $\mathbf{a}, \mathbf{b}$ and $\mathbf{f}$ are vectors with $a_{n}, b_{n}$ and $f_{n}$ as components respectively, and $\mathbf{Q}$ is a matrix with elements $Q_{m n}$. We remark here that what was important for us here was the existence of $\mathbf{S}_{\mathbf{1}}$ and $\mathbf{S}_{\mathbf{2}}$ not their particular radii, however, we will see below that a small condition number in the sense that these radii are close give a fast convergence for the numerical method.

Note that the matrix $\mathbf{Q}$ is of infinite-dimensional, in numerical simulation, it is truncated to finite-dimensional matrix.

We remark that if we want to compute the scattered field from another point source we need only recompute a using (10) while $\mathbf{Q}$ is not affected. At the internal resonance of the cavity formed by $\mathbf{S}$, the matrix $\mathbf{Q}$ is ill-conditioned. This problem can be overcome by using a complete set to expand the surface sources, see [11] for details. Here, we exclude situations with internal resonances for simplicity.

We now define the scattering matrix $\mathbf{T}$ that relate the scattered wave amplitude to the incoming wave amplitude

$$
\begin{gathered}
\mathbf{T}=-\operatorname{Real}[\mathbf{Q}] \cdot \mathbf{Q}^{-1} \\
\mathbf{f}=\mathbf{T} \cdot \mathbf{a} .
\end{gathered}
$$

Note that once the scattering matrix $\mathbf{T}$ for one target is found, it can be used collectively with the scattering matrices of the other scatterers to construct recursively the scattered wave field by many targets [11].

The EBCM is also known as the null-field approach, and the convergence has been studied in $[1,2]$. 


\section{The Coupled Scattering System}

We finally consider multiple scattering between the extended target and the point scatterers. In this case we can write the scattered field as

$$
\phi_{s c a}(\mathbf{r})=\sum_{j=1}^{N} \sigma_{j} g\left(\mathbf{r}, \mathbf{r}_{j}\right) \phi_{j}^{E}-\int_{\mathbf{S}} d s^{\prime} \hat{n} \cdot\left[g\left(\mathbf{r}, \mathbf{r}^{\prime}\right) \nabla^{\prime} \phi^{E}\left(\mathbf{r}^{\prime}\right)\right], \quad \mathbf{r} \in R^{2} \backslash D
$$

where we denoted the exciting 'fields' at the point scatterers by $\phi_{j}^{E}$ and at the extended scatterer by $\phi^{E}\left(\mathbf{r}^{\prime}\right)$ respectively. Below we shall obtain a coupled infinite dimensional system for the exciting field at the point scatterers and the coefficients in a cylindrical harmonics expansion of the exciting field at the extended scatterer. The numerical implementation in the Section 5 is based on a finite dimensional approximation of this system.

For $|\mathbf{r}|>\max _{j}\left|\mathbf{r}_{j}\right|$ we write the scattered field by all scatterers as

$$
\psi_{\text {out }}^{F}=\sum_{n} \phi_{\text {out }}^{F}(n) S_{n}(\mathbf{r})
$$

and the incoming field due to the source by

$$
\psi_{i n c}^{F}=\sum_{n} \phi_{i n c}^{F}(n) R_{n}(\mathbf{r})
$$

We now assume

Assumption $4.1 \min _{j}\left|\mathbf{r}_{j}\right|>\max _{\mathbf{r} \in \mathbf{S}}|\mathbf{r}|$.

This means that we can define a non-empty annulus outside of the extended scatterer

and inside of the point scatterers. For $\mathbf{r}$ such that $\left(\max \left|\mathbf{r}^{\prime}\right|\right.$ for $\left.\mathbf{r}^{\prime} \in \mathbf{S}\right)<|\mathbf{r}|<\min _{j}\left|\mathbf{r}_{j}\right|$ we write the incoming field due to the source and scattering off the point scatterers as

$$
\psi_{i n c}^{N}=\sum_{n} \phi_{i n c}^{N}(n) R_{n}(\mathbf{r}),
$$




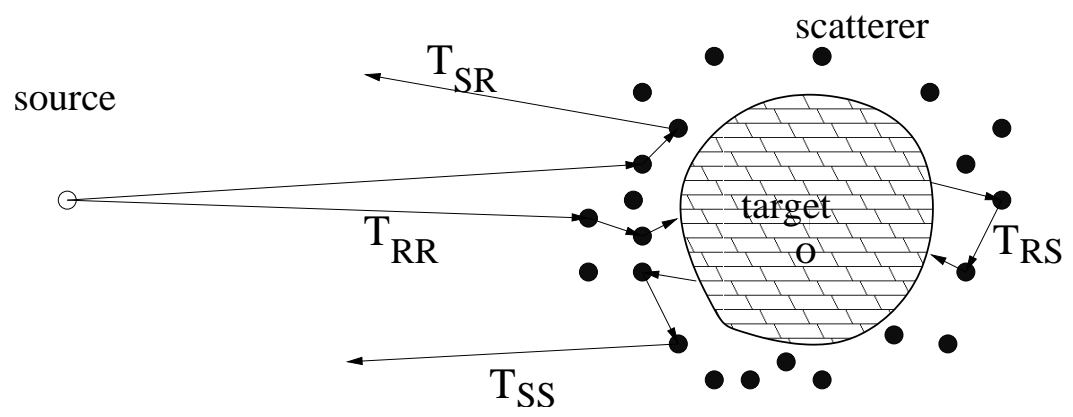

Figure 3: Multiple Scattering Operators

and the field scattered by the extended scatter as

$$
\psi_{\text {out }}^{N}=\sum_{n} \phi_{\text {out }}^{N}(n) S_{n}(\mathbf{r}) .
$$

We now introduce the linear operators :

$$
\begin{aligned}
\mathbf{T}_{R R} & =\mathbf{I}+\mathbf{A Z}^{-1} \mathbf{C}, \\
\mathbf{T}_{S S} & =\mathbf{I}+\mathbf{H Z}^{-1} \mathbf{P}, \\
\mathbf{T}_{R S} & =\mathbf{A} \mathbf{Z}^{-1} \mathbf{P}, \\
\mathbf{T}_{S R} & =\mathbf{H Z}^{-1} \mathbf{C}, \\
\mathbf{T}_{X} & =-\operatorname{Real}(\mathbf{Q}) \mathbf{Q}^{-1},
\end{aligned}
$$

with

$$
A_{n j}=i \sigma_{j} S_{n}\left(\mathbf{r}_{j}\right), \quad H_{n j}=i \sigma_{j} R_{n}\left(\mathbf{r}_{j}\right), \quad C_{j n}=R_{n}\left(\mathbf{r}_{j}\right), \quad P_{j n}=S_{n}\left(\mathbf{r}_{j}\right) .
$$

We then have the system

$$
\left[\begin{array}{c}
\boldsymbol{\phi}_{\text {inc }}^{N} \\
\boldsymbol{\phi}_{\text {out }}^{F}
\end{array}\right]=\left[\begin{array}{ll}
T_{R R} & T_{R S} \\
T_{S R} & T_{S S}
\end{array}\right]\left[\begin{array}{c}
\boldsymbol{\phi}_{i n c}^{F} \\
\boldsymbol{\phi}_{\text {out }}^{N}
\end{array}\right],
$$

moreover, we have

$$
\phi_{o u t}^{N}=T_{X} \phi_{i n c}^{N} \text {. }
$$


This gives then

$$
\phi_{i n c}^{N}=T_{R R} \phi_{i n c}^{F}+T_{R S} T_{X} \phi_{i n c}^{N}
$$

so that

$$
\begin{aligned}
& \phi_{i n c}^{N}=\left(I-T_{R S} T_{X}\right)^{-1} T_{R R} \phi_{i n c}^{F} \\
& \phi_{\text {out }}^{F}=\left(T_{S R}+T_{S S} T_{X}\left(I-T_{R S} T_{X}\right)^{-1} T_{R R}\right) \phi_{i n c}^{F} .
\end{aligned}
$$

We give the detailed derivation in Appendix A.

\section{$5 \quad$ Numerical Examples}

In this section, we will show four numerical examples. We used 31 terms in the wave function expansions, $n=-15, \cdots, 15$, in these simulation. Consequently, the size of matrix $Q$ is $31 \times 31$. With $n=15$, the error in the expansion in (9) is less than $10^{-10}$ for our test examples.

The wavelength is chosen as the unit in our examples and figures. We used two types of extended target. The first target is a disk, the second is a half disk plus a cone, see Figure 4. The radius of disk is 3 wavelengths.

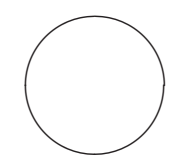

Target 1

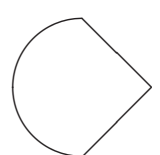

Target 2

Figure 4: Geometries of the extended targets.

In the first example in Figure 5, the extended target is the disk. The source is 12 wavelength away from the left side of the target. We put one point scatterer on top 
of the target (3 wavelengths away). The left figure plots then the wave field, while the right figure plots the difference wave field with or without the point scatterer. We see clearly that the difference field contains strong reflections from the top part of the target due to multiple scattering between the point scatterer and the extended target. This information can be used to image the top part of the target.

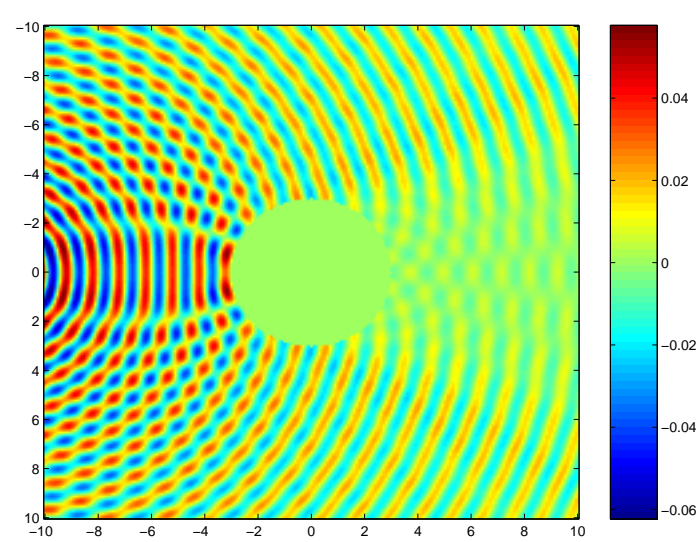

(1) Wave field

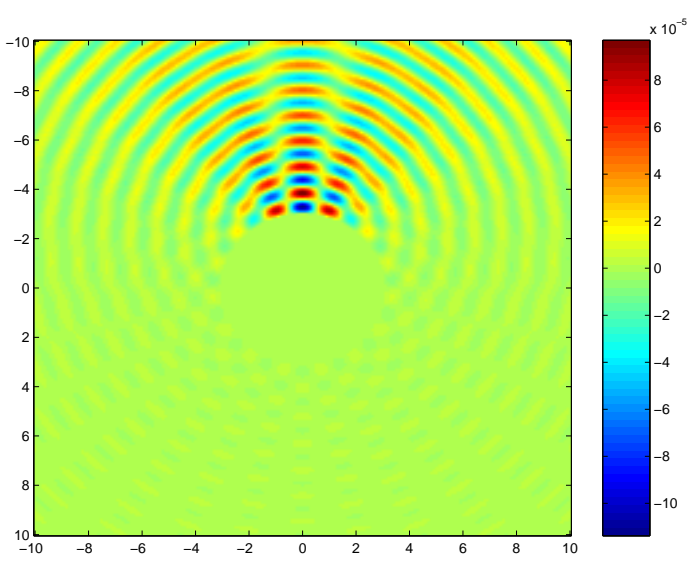

(2) The difference field

Figure 5: Example 1; Target 1 and one point scatterer.

In the second example, in Figure 6, the setting is as before, but the extended target is the Target 2. We remark that we do not evaluate the wave field inside the disk since we are interested in the scattered field. We see that the difference field has a different pattern from Example 1. This phenomenon can be exploited for classification purposes.

In the third example, in Figure 7, we randomly put 200 point scatterers surrounding the Target 2. In this case the difference field corresponding to specular reflection from the target and scattering from the tip of the cone (as in the previous example) is relatively strongest. We remark that with our approach we can easily handle a large number of point scatterers, e.g. thousands.

In the last example, in Figure 8, the source is 1000 wavelengths away from the Target 2. There are 10 random point scatterers surrounding the target. Note how 


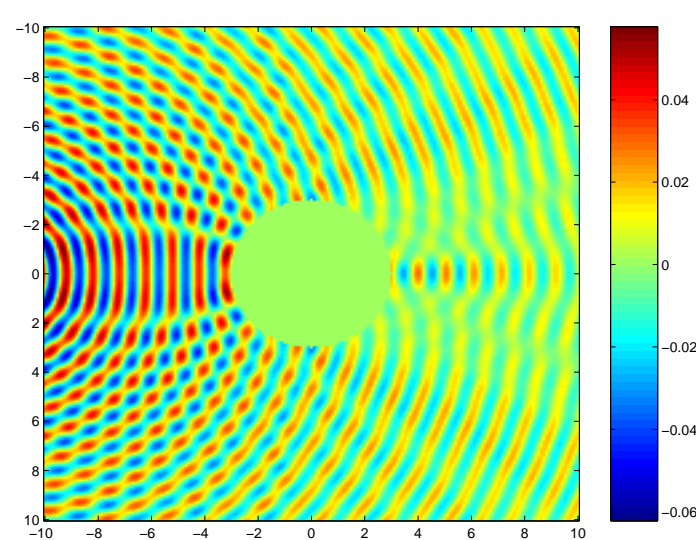

(1) Wave field

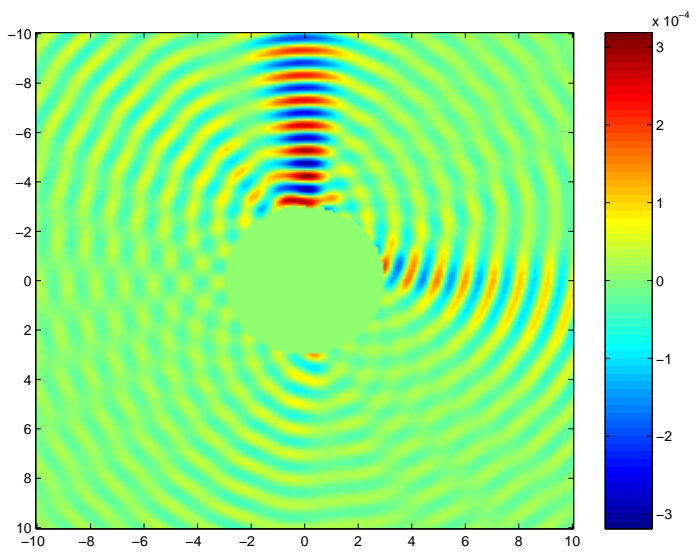

(2) Difference field

Figure 6: Example 2; Target 2 and one point scatterer.

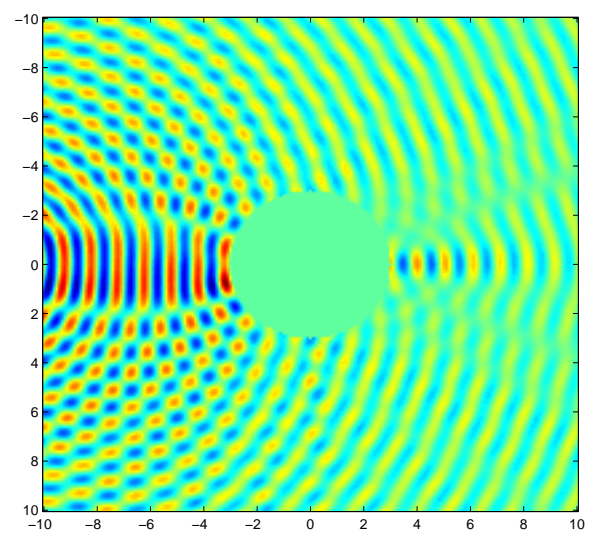

(1) Wave field
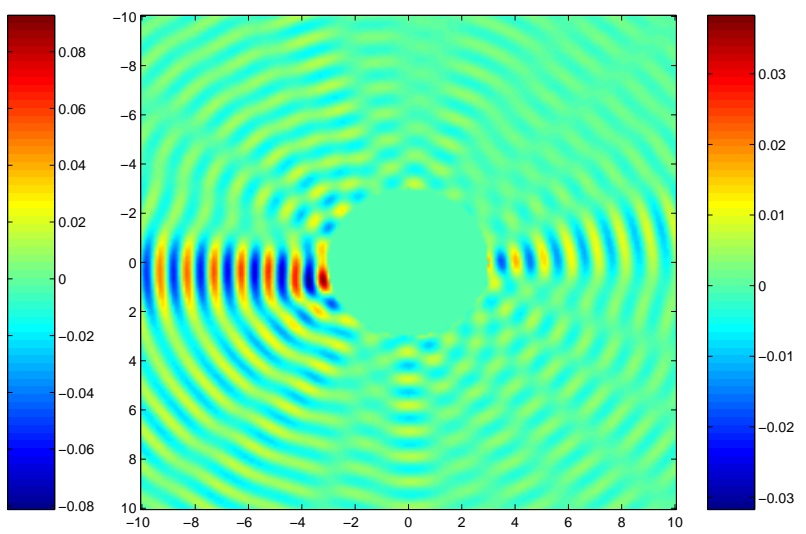

(2) Difference field

Figure 7: Example 3; Target 2 and 200 point scatterers. 
the difference field gives information about the location of the point targets and also information about the geometry of the extended target via the point scatterers. The example illustrates that we easily can resolve far field phenomena. The main computation is the formation of the matrix $Q$, in which we used adaptive Simpson quadrature.

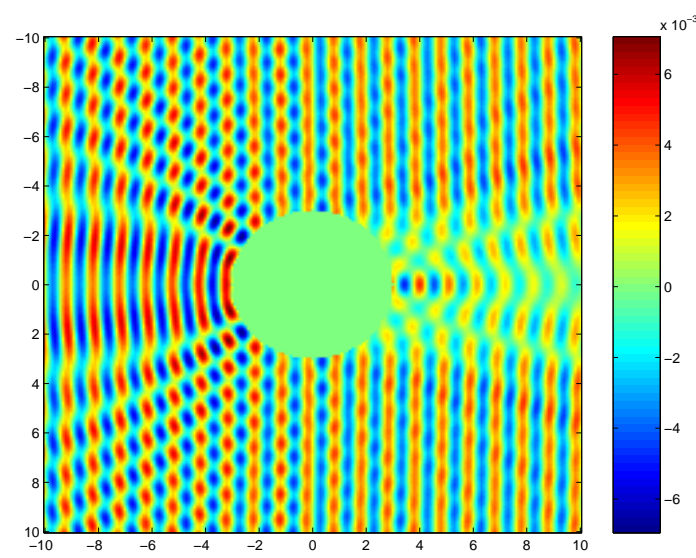

(1) a. Wave field

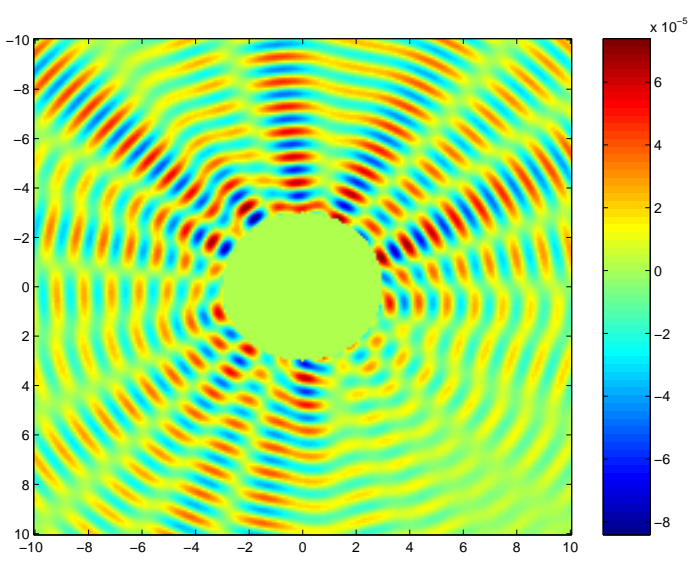

(2) b. Difference field

Figure 8: Example 4. Source in far field.

We conclude that we have been able to calculate the scattered fields in the FoldyLax formulation in the presence of an extended scatterer, a situation corresponding to a strong separation of scales and where using discretization of the Helmholtz equation would have been very expensive.

\section{Conclusion}

We have developed an efficient numerical algorithm to simulate multiple scattering among point scatterers and extended targets in 2D. In our algorithm the free space Green's function is used and no partial differential equations need to be discretized and solved. The main computational cost is the formation of the matrix $Q$. In our approach we can easily deal with long propagation distances and large number of point scatterers. Extension to 3D is being studied. 


\section{A Generalized Foldy Lax System}

In this appendix we derive the generalized Foldy Lax formulation that gives the exciting

field at the extended scatterer and the point scatterer. The excited field can then be used to compute the scattered field at any point in the domain.

Let the exciting field at the scatterers be denoted by

$$
\phi_{j}^{E}=\phi\left(\mathbf{r}_{j}\right), \quad 1 \leq j \leq N
$$

The impinging (far field) source field may for instance be generated by a set of point sources. We write it as in (19) :

$$
\psi_{i n c}^{F}(\mathbf{r})=\sum_{n} \phi_{i n c}^{F}(n) R_{n}(\mathbf{r}) .
$$

The incoming field on $\mathbf{S}_{2}$, see Figure 3 , is now

$$
\begin{aligned}
\phi_{i n c}(\mathbf{r}) & =\sum_{n=-\infty}^{\infty} R_{n}(\mathbf{r}) \phi_{i n c}^{F}(n)+\sum_{j=1}^{N} \sigma_{j} \phi_{j}^{E} g\left(\mathbf{r}, \mathbf{r}_{j}\right) \\
& =\sum_{n=-\infty}^{\infty} R_{n}(\mathbf{r}) \phi_{i n c}^{F}(n)+i \sum_{j=1}^{N} \sigma_{j} \phi_{j}^{E} \sum_{n=-\infty}^{\infty} S_{n}\left(\mathbf{r}_{j}\right) R_{n}(\mathbf{r}) \\
& =\sum_{n=-\infty}^{\infty}\left(\phi_{i n c}^{F}(n)+\sum_{j=1}^{N} A_{n j} \phi_{j}^{E}\right) R_{n}(\mathbf{r}) \\
& =\mathbf{R}(\mathbf{r}) \cdot\left(\phi_{i n c}^{F}+\mathbf{A} \phi^{E}\right)
\end{aligned}
$$

with

$$
A_{n j}=i \sigma_{j} S_{n}\left(\mathbf{r}_{j}\right)
$$

We then use (7) and (11) to find for $\mathbf{r} \in \mathbf{S}_{2}$

$$
\begin{aligned}
\left(\phi_{i n c}^{F}+\mathbf{A} \phi^{E}\right) \cdot \mathbf{R}(\mathbf{r}) & =i \sum_{m} b_{m} \int_{\mathbf{S}} d s^{\prime} \sum_{n=-\infty}^{\infty}\left[S_{n}\left(\mathbf{r}^{\prime}\right) \hat{n} \cdot \nabla^{\prime} R_{m}\left(\mathbf{r}^{\prime}\right)\right] R_{n}(\mathbf{r}) \\
& =i \mathbf{Q b} \cdot \mathbf{R}(\mathbf{r})
\end{aligned}
$$


and in view of the orthogonality of the cylindrical harmonics

$$
\phi_{i n c}^{F}+\mathbf{A} \phi^{E}=i \mathbf{Q b}
$$

which generalizes the relation (12).

Next, let $|\mathbf{r}|>\max _{\mathbf{r} \in \mathbf{S}}|\mathbf{r}|$ and write the field scattered by the extended scatterer as

$$
\phi_{s c a}(\mathbf{r})=\sum_{n=-\infty}^{\infty} f_{n}^{X} S_{n}(\mathbf{r})
$$

then we have as in (15)

$$
\mathbf{f}^{\mathbf{X}}=-i \operatorname{Real}(\mathbf{Q}) \mathbf{b}
$$

with $\mathbf{b}$ defined as in (11). In view of (31) we then find

$$
\mathbf{f}^{\mathbf{X}}=-\operatorname{Real}(\mathbf{Q}) \mathbf{Q}^{-1}\left(\phi_{i n c}^{F}+\mathbf{A} \phi^{E}\right)
$$

The exciting field at the scatterers can now be identified as

$$
\phi_{j}^{E}=\mathbf{R}\left(\mathbf{r}_{j}\right) \cdot \phi_{i n c}^{F}+\sum_{l=1 \neq j}^{N} \sigma_{l} g\left(\mathbf{r}_{j}, \mathbf{r}_{l}\right) \phi_{l}^{E}+\sum_{n=-\infty}^{\infty} f_{n}^{X} S_{n}\left(\mathbf{r}_{j}\right), \quad 1 \leq j \leq N .
$$

We write these relations in the form

$$
\mathbf{Z} \phi^{E}=\mathbf{C} \phi_{i n c}^{F}+\mathbf{P f}^{\mathbf{X}}
$$

for $\mathbf{Z}$ defined in (3) and

$$
C_{j n}=R_{n}\left(\mathbf{r}_{j}\right), \quad P_{j n}=S_{n}\left(\mathbf{r}_{j}\right)
$$

From (32) and (33) we then find

$$
\mathbf{f}^{\mathbf{X}}=-\operatorname{Real}(\mathbf{Q}) \mathbf{Q}^{-1}\left(\phi_{i n c}^{F}+\mathbf{A} \mathbf{Z}^{-1}\left(\mathbf{C} \phi_{i n c}^{F}+\mathbf{P f}^{\mathbf{X}}\right)\right)
$$

so that

$$
\mathbf{f}^{\mathbf{X}}=\left[\mathbf{I}+\operatorname{Real}(\mathbf{Q}) \mathbf{Q}^{-1} \mathbf{A} \mathbf{Z}^{-1} \mathbf{P}\right]^{-1}\left(-\operatorname{Real}(\mathbf{Q}) \mathbf{Q}^{-1}\left(\mathbf{I}+\mathbf{A Z}^{-1} \mathbf{C}\right)\right) \boldsymbol{\phi}_{i n c}^{F}
$$


Recall the operators introduced in Figure 3 and below:

$$
\begin{aligned}
\mathbf{T}_{R R} & =\mathbf{I}+\mathbf{A Z}^{-1} \mathbf{C}, \\
\mathbf{T}_{X} & =-\operatorname{Real}(\mathbf{Q}) \mathbf{Q}^{-1}, \\
\mathbf{T}_{R S} & =\mathbf{A Z}^{-1} \mathbf{P},
\end{aligned}
$$

then we have

$$
\mathbf{f}^{\mathbf{X}}=\left[\mathbf{I}-\mathbf{T}_{X} \mathbf{T}_{R S}\right]^{-1} \mathbf{T}_{X} \mathbf{T}_{R R} \phi_{i n c}^{F}=\mathbf{T}_{X}\left[\mathbf{I}-\mathbf{T}_{R S} \mathbf{T}_{X}\right]^{-1} \mathbf{T}_{R R} \boldsymbol{\phi}_{i n c}^{F} .
$$

Finally, we derive the expression for the scattered field that is generated by the exciting field at both the point scatterers and the extended scatterer. Let the point of field evaluation, $\mathbf{r}$, be such that $|\mathbf{r}|>\max _{j}\left|\mathbf{r}_{j}\right|$. The field generated by the exciting field at the scatters is

$$
\begin{array}{r}
\phi_{\text {sca }}^{P}=\sum_{j=1}^{N} \phi_{j}^{E} \sigma_{j} g\left(\mathbf{r}, \mathbf{r}_{j}\right)=\sum_{j=1}^{N} \phi_{j}^{E} \sigma_{j} \sum_{n=-\infty}^{\infty} i S_{n}(\mathbf{r}) R_{n}\left(\mathbf{r}_{j}\right) \\
=\sum_{n=-\infty}^{\infty}\left(\sum_{j=1}^{N} H_{n j} \phi_{j}^{E}\right) S_{n}(\mathbf{r}) \equiv \sum_{n=-\infty}^{\infty} f_{n}^{P} S_{n}(\mathbf{r}),
\end{array}
$$

for

$$
H_{n j}=i \sigma_{j} R_{n}\left(\mathbf{r}_{j}\right)
$$

The total scattered wave field coefficients are then

$$
\begin{aligned}
& \mathbf{f}^{\mathbf{X}}+\mathbf{f}^{P} \\
= & \left\{\mathbf{T}_{X}\left[\mathbf{I}-\mathbf{T}_{R S} \mathbf{T}_{X}\right]^{-1} \mathbf{T}_{R R} \phi_{i n c}^{F}+\mathbf{H Z}^{-1}\left(\mathbf{C} \phi_{i n c}^{F}+\mathbf{P f} \mathbf{X}^{\mathbf{X}}\right)\right\} \\
= & \left\{\mathbf{T}_{X}\left[\mathbf{I}-\mathbf{T}_{R S} \mathbf{T}_{X}\right]^{-1} \mathbf{T}_{R R}+\mathbf{H Z}^{-1} \mathbf{C}+\mathbf{H Z}^{-1} \mathbf{P} \mathbf{T}_{X}\left[\mathbf{I}-\mathbf{T}_{R S} \mathbf{T}_{X}\right]^{-1} \mathbf{T}_{R R}\right\} \boldsymbol{\phi}_{i n c}^{F} .
\end{aligned}
$$

We again use a notation as in Figure 3:

$$
\begin{aligned}
& \phi_{\text {out }}^{F}=\mathbf{f}^{\mathbf{X}}+\mathbf{f}^{P}, \\
& \mathbf{T}_{S S}=\mathbf{I}+\mathbf{H Z}^{-1} \mathbf{P}, \quad \mathbf{T}_{S R}=\mathbf{H Z}^{-1} \mathbf{C},
\end{aligned}
$$


to obtain

$$
\phi_{\text {out }}^{F}=\left(\mathbf{T}_{S R}+\mathbf{T}_{S S} \mathbf{T}_{X}\left[\mathbf{I}-\mathbf{T}_{R S} \mathbf{T}_{X}\right]^{-1} \mathbf{T}_{R R}\right) \phi_{\text {inc }}^{F}
$$

which is (30).

We finally turn our interest to the total field impinging on the extended scatterer. We write for $\mathbf{r}<\min _{j}\left|\mathbf{r}_{j}\right|$

$$
\phi_{i n c}(\mathbf{r})=\sum_{n=-\infty}^{\infty} \phi_{i n c}^{N}(n) R_{n}(\mathbf{r})
$$

and have then

$$
\begin{aligned}
\phi_{i n c}^{N} & =\left(\phi_{i n c}^{F}+\mathbf{A} \phi^{E}\right) \\
& =\left(\phi_{i n c}^{F}+\mathbf{A} \mathbf{Z}^{-1} \mathbf{C} \phi_{i n c}^{F}+\mathbf{A} \mathbf{Z}^{-1} \mathbf{P} \mathbf{T}_{X}\left[\mathbf{I}-\mathbf{T}_{R S} \mathbf{T}_{X}\right]^{-1} \mathbf{T}_{R R} \boldsymbol{\phi}_{i n c}^{F}\right) \\
& =\left(\mathbf{T}_{R R}+\mathbf{T}_{R S} \mathbf{T}_{X}\left[\mathbf{I}-\mathbf{T}_{R S} \mathbf{T}_{X}\right]^{-1} \mathbf{T}_{R R}\right) \phi_{i n c}^{F} \\
& =\left[\mathbf{I}-\mathbf{T}_{R S} \mathbf{T}_{X}\right]^{-1} \mathbf{T}_{R R} \phi_{i n c}^{F}
\end{aligned}
$$

which is $(29)$.

\section{References}

[1] R.H.T. Bates and D.J.N. Wall, Null field approach to scalar diffraction, I. General methods, II. Approximation methods, Phil. Trans. R. Soc. Lond. A287:45-95, 1977.

[2] J.C. Bolomey and A. Wirgin, Numerical comparison of the Green's function and the Waterman and Rayleigh theories of scattering from a cylinder, Proc. IEE 121: 794-804, 1974.

[3] M. Born and E. Wolf, Principles of Optics. Academic Press, New York. 1970.

[4] D. Colton and R. Kress, Inverse Acoustic and Electromagnetic Scattering Theory, Springer, 1998. 
[5] L.L. Foldy, The multiple scattering of waves, Pyhs. Rev. 67, 107-119, 1945.

[6] M. Lax, Multiple scattering of waves, Rev. Modern. Phys., 23, 287-310, 1951.

[7] M. Lax, Multiple scattering of waves II, The effective field in dense systems, Phys, Rev., 85, 261-269, 1952.

[8] Leung Tsang, Jin-Au Kong, Kung-Hau Ding and Chi-On Ao, Scattering of electromagnetic waves, Numerical Simulations, John Wiley \& Sons, Inc., New York, 2001.

[9] P.C. Waterman, New Formulation of acoustic scattering, J. Acoust. Soc. Am. 45: 1417-1429, 1969.

[10] P.C. Waterman, Symmetry, unitarity and geometry in electromagnetic scattering, Phys. Rev. D 3(4): 825-829, 1971.

[11] Weng Cho Chew, Waves and Fields in Inhomogeneous Media, Van Nostrand Reinhold, New York, 1990. 